\title{
Dynamical Analysis of a Stochastic Predator-Prey Model with an Allee Effect
}

\author{
Feng Rao \\ College of Sciences, Nanjing University of Technology, Nanjing 211816, China \\ Correspondence should be addressed to Feng Rao; raofeng2002@163.com
}

Received 23 July 2013; Revised 24 October 2013; Accepted 7 November 2013

Academic Editor: Chengjian Zhang

Copyright ( 2013 Feng Rao. This is an open access article distributed under the Creative Commons Attribution License, which permits unrestricted use, distribution, and reproduction in any medium, provided the original work is properly cited.

We present and analyze a modified Holling type-II predator-prey model that includes some important factors such as Allee effect, density-dependence, and environmental noise. By constructing suitable Lyapunov functions and applying Itô formula, some qualitative properties are given, such as the existence of global positive solutions, stochastic boundedness, and the global asymptotic stability. A series of numerical simulations to illustrate these mathematical findings are presented.

\section{Introduction}

The study of the interrelationship between species and their environment, ecology, in such areas as predator-prey and competition interactions, renewable resource management, evolution of pesticide resistant strains, ecological and genetically engineered control of pests, multispecies societies, and so on, is now an enormous field [1]. Predator-prey interaction is one of the basic interspecies relations for ecological and social models, and it is also a basic block of more complicated food chain, food web and biochemical network structure [2]. In recent years, one of the important predator-prey models is the Holling type-II model, originally due to Holling [3, 4], which has been extensively studied in many articles [5-11].

In ecological populations, species growth is often damped by an Allee effect. The so-called Allee effect is described by the positive relationship between population density and the per capita growth rate $[12,13]$. The logistic model assumes that per capita growth rate declines monotonically with density. But for population subject to an Allee effect, per capita growth rate shows a humped curve increasing (from negative to positive) at low density, up to a maximum at intermediate density and then declining [14]. The Allee effect may arise from a number of sources such as difficulties in finding mates when population density is low, social dysfunction at small population sizes, food exploitation, inbreeding depression, and predator avoidance or defense [15]. This effect usually saturates or disappears as population get larger [12]. The Allee effect has been modeled in different ways [16-18], such as multiplicative Allee effect and additive Allee effect. In an ecological point of view, Allee effect has been modeled into strong and weak cases. The strong Allee effect introduces a population threshold, and the population must surpass this threshold to grow. In contrast, population with a weak Allee effect do not have a threshold [19]. the Allee effect's strong potential impact on population dynamics has been attracting much attention recently. Detailed investigations relating to the Allee effect may be found in [12-15, 17-28].

In reality, there are many benefits to be gained in using stochastic models because real life is full of randomness and stochasticity. A large number of stochastic differential equation models are considered as they provide some additional degree of realism compared to their deterministic counterpart [2]. The environment factors are time dependent randomly varying and should be taken as stochastic $[29,30]$. The basic mechanism and factors of population growth like the resources and vital rates-birth, death, immigration and emigration change nondeterministically due to random environment [31]. Most natural phenomena do not follow strictly deterministic laws but rather oscillate randomly about some average values, so that the population density never attains a fixed value with the advancement of time [32]. In predator-prey model, the random fluctuations are also undeniably arising from either environmental variability or internal species. For example, the birth and death processes 
of individuals are intrinsically stochastic fluctuations which become especially pronounced when the number of individuals is small [33]. Recent advances in stochastic differential equations enable a lot of authors to introduce stochastic factor into the model of physical phenomena, whether it is a random noise in the system of differential equations or environmental fluctuations in parameters [34-45].

Based upon these factors, to the best of our knowledge, a small amount of work has been done with stochastic perturbation on a modified Holling type-II predator-prey model which has an Allee effect on prey population and density-dependence of predator population. The purpose of this paper is to study that the stochastic factor has a significant effect on the dynamics of Holling type-II predatorprey model with an Allee effect and density-dependence. The organization of this paper is as follows. In the next section, we present the formulation of mathematical model with Allee effect, density-dependence, and environmental noise. Moreover, we present a qualitative analysis of the stochastic model. In Section 3, we use numerical simulations to reveal the influence of noise on the dynamical behavior of the model. A brief discussion is given in Section 4 .

\section{The Model and Analysis}

In this paper, we present a Holling type-II predator-prey model that includes some important factors such as an Allee effect on prey population, density-dependence of predator population, and environmental noise. Assuming that the random factors in the environment would display themselves mainly as fluctuations on the intrinsic growth rates of prey and predator, then the stochastic modified Holling type-II predation model takes the following form:

$$
\begin{gathered}
d N=N\left(1-N-\frac{m}{b+N}-\frac{w P}{a+N}\right)\left(r d t+\alpha d \eta_{1}(t)\right) \\
d P=P\left(\frac{N}{a+N}-d-c P\right)\left(s d t+\beta d \eta_{2}(t)\right) \\
N(0)=N_{0}>0, \quad P(0)=P_{0}>0
\end{gathered}
$$

Here, we replace the intrinsic growth rates $r(>0)$ and $s$ $(>0)$ of prey and predator by

$$
r \longrightarrow r+\alpha \dot{\eta}_{1}(t), \quad s \longrightarrow s+\beta \dot{\eta}_{2}(t) .
$$

In the above model, $N=N(t)$ and $P=P(t)$ represent prey and predator densities at time $t$, respectively. $m N /(b+N)$ is the term of additive Allee effect and $m \in(0,1)$ and $b \in$ $(0,1)$ are Allee effect constants. If $m<b$, the function of prey growth rate with the Allee effect of the prey is called weak Allee effect; if $m>b$, the modified function of prey growth rate is strong Allee effect. $c P$ stands for the densitydependence of predator population and $c>0 . w(>0)$ is the predation rate, $a(>0)$ the half-saturation density of Holling type-II functional response, and $d(>0)$ the predator death rate. The positive constants $\alpha$ and $\beta$, respectively, are the coefficients of the effect of the environmental stochastic perturbation on the prey and on the predator population. $\dot{\eta}_{i}(t)(i=1,2)$ is a standard white noise with increment $\Delta \eta_{i}(t)=\eta_{i}(t+\Delta t)-\eta_{i}(t)$ that is independent Gaussian random variables $\mathbf{N}(0, \Delta t)$. That is to say, $\eta_{i}(t)(i=1,2)$ is a Brownian motion defined on a complete probability space $(\Omega, \mathscr{F}, \mathbf{P})$ with a filtration $\left\{\mathscr{F}_{t}\right\}_{t \in \mathbb{R}_{+}}$satisfying the usual conditions; namely, it is right continuous and increasing while $\mathscr{F}_{0}$ contains all $\mathbf{P}$-null sets [46].

Consider model (1) as an Itô stochastic differential system of the type

$$
d U(t)=F(t, U(t)) d t+G(t, U(t)) d \eta(t), \quad U\left(t_{0}\right)=U_{0},
$$

where

$$
\begin{gathered}
U(t)=(N(t), P(t))^{T}, \quad \eta(t)=\left(\eta_{1}(t), \eta_{2}(t)\right)^{T}, \\
F=\left(\begin{array}{c}
r N\left(1-N-\frac{m}{b+N}-\frac{w P}{a+N}\right) \\
s P\left(\frac{N}{a+N}-d-c P\right)
\end{array}\right), \\
G=\left(\begin{array}{cc}
\alpha N\left(1-N-\frac{m}{b+N}-\frac{w P}{a+N}\right) & 0 \\
0 & \beta P\left(\frac{N}{a+N}-d-c P\right)
\end{array}\right),
\end{gathered}
$$

and the solution $U(t)(t>0)$ is an Itô process, $F$ is a slowly varying continuous component or drift coefficient, and $G$ is the rapidly varying continuous random component or diffusion coefficient [46].

2.1. Existence of Global Positive Solutions. For a stochastic differential equation, in order to have a unique global solution (i.e., no explosion in a finite time) for any given initial value, the coefficients of the equation are generally required to satisfy the linear growth condition and local Lipschitz condition [46]. To show that model (1) has a global positive solution, let us firstly prove that the model has a positive local solution by making the change of variables.

Theorem 1. There is a unique local solution $(N(t), P(t))$ for $t \in\left[0, \tau_{e}\right)$ to model (1) a.s. for any given initial value $\left(N_{0}, P_{0}\right) \in$ $\mathbb{R}_{+}^{2}=\left\{N, P \in \mathbb{R}^{2} \mid N>0, P>0\right\}$, where $\tau_{e}$ is the explosion time.

Proof. Set $p(t)=\ln N(t), q(t)=\ln P(t)$ and we consider the equations

$$
\begin{aligned}
& d p= {\left[r\left(1-e^{p}-\frac{m}{b+e^{p}}-\frac{w e^{q}}{a+e^{p}}\right)\right.} \\
&\left.-\frac{\alpha^{2}}{2}\left(1-e^{p}-\frac{m}{b+e^{p}}-\frac{w e^{q}}{a+e^{p}}\right)^{2}\right] d t \\
&+\alpha\left(1-e^{p}-\frac{m}{b+e^{p}}-\frac{w e^{q}}{a+e^{p}}\right) d \eta_{1}(t),
\end{aligned}
$$




$$
\begin{gathered}
d q=\left[s\left(\frac{e^{p}}{a+e^{p}}-d-c e^{q}\right)-\frac{\beta^{2}}{2}\left(\frac{e^{p}}{a+e^{p}}-d-c e^{q}\right)^{2}\right] d t \\
+\beta\left(\frac{e^{p}}{a+e^{p}}-d-c e^{q}\right) d \eta_{2}(t), \\
p(0)=\ln N_{0}, \quad q(0)=\ln P_{0}
\end{gathered}
$$

on $t \geq 0$. Then we know that the coefficients of (2.1) satisfy the local Lipschitz condition, and there is a unique local solution $(p(t), q(t))$ on $\left[0, \tau_{e}\right)$. By Itô's formula, we can see that $N(t)=$ $e^{p(t)}, P(t)=e^{q(t)}$ is the unique positive local solution to model (1) with the initial value $N_{0}>0, P_{0}>0$.

Next, we show that the solution of model (1) is global; that is, $\tau_{e}=\infty$ a.s.

Theorem 2. For model (1) and any given initial value $\left(N_{0}, P_{0}\right) \in \mathbb{R}_{+}^{2}$, there is a unique solution $(N(t), P(t))$ on $t \geq 0$ and the solution will remain in $\mathbb{R}_{+}^{2}$ with probability one.

Proof. Let $n_{0}>0$ be sufficiently large for $N_{0}$ and $P_{0}$ lying within the interval $\left[1 / n_{0}, n_{0}\right]$. For each integer $n \geq n_{0}$, let us define a sequence of stopping times by

$$
\tau_{n}=\inf \left\{t \in\left[0, \tau_{e}\right]: N(t) \notin\left(\frac{1}{n}, n\right) \text { or } P(t) \notin\left(\frac{1}{n}, n\right)\right\},
$$

where we set $\inf \emptyset=\infty$ ( $\emptyset$ represents the empty set) in this paper. Since $\tau_{n}$ is nondecreasing as $n \rightarrow \infty$, there exists the limit

$$
\tau_{\infty}=\lim _{n \rightarrow \infty} \tau_{n} .
$$

Then

$$
\tau_{\infty} \leq \tau_{e} \text { a.s. }
$$

Now, we need to show $\tau_{\infty}=\infty$ a.s. If this statement is violated, then there exist $T>0$ and $\varepsilon \in(0,1)$ such that

$$
\mathbf{P}\left\{\tau_{\infty} \leq T\right\}>\varepsilon
$$

Thus, there is an integer $n_{1} \geq n_{0}$ such that

$$
\mathbf{P}\left\{\tau_{n} \leq T\right\} \geq \varepsilon \quad \forall n \geq n_{1} .
$$

Define a $\mathbb{C}^{2}$-function $V: \mathbb{R}_{+}^{2} \rightarrow \mathbb{R}_{+}$by

$$
V(N, P)=N+P-\ln N P-2,
$$

which is a nonnegative function. If $(N(t), P(t)) \in \mathbb{R}_{+}^{2}$, by using Itô's formula, we compute

$$
\begin{aligned}
d V(N, P)=[ & 2 r(N-1)\left(1-N-\frac{m}{b+N}-\frac{w P}{a+N}\right) \\
& +2 s(P-1)\left(\frac{N}{a+N}-d-c P\right) \\
& +\frac{\alpha^{2}}{2}\left(1-N-\frac{m}{b+N}-\frac{w P}{a+N}\right)^{2} \\
& \left.+\frac{\beta^{2}}{2}\left(\frac{N}{a+N}-d-c P\right)^{2}\right] d t
\end{aligned}
$$

$$
\begin{aligned}
& +\alpha(N-1)\left(1-N-\frac{m}{b+N}-\frac{w P}{a+N}\right) d \eta_{1} \\
& +\beta(P-1)\left(\frac{N}{a+N}-d-c P\right) d \eta_{2} \\
& \leq\left[2 r(1-N)\left(\frac{m}{b+N}+\frac{w P}{a+N}\right)\right. \\
& \left.+\frac{\alpha^{2}}{2}\left(1-N-\frac{m}{b+N}-\frac{w P}{a+N}\right)^{2}\right] d t \\
& +\left[2 s\left(\frac{N P}{a+N}+d+c P\right)\right. \\
& +\beta(P-1)\left(\frac{N}{a+N}-d-c P\right) d \eta_{2}, \\
& +\alpha\left(\frac{\beta^{2}}{2}\left(\frac{N}{a+N}-d-c P\right)^{2}\right] d t \\
& +\beta(P-1)\left(\frac{N}{a+N}-d-c P\right) d \eta_{2} \\
& \times M_{1}+M_{2}+\alpha(N-1) \\
& \left.+N-\frac{m}{b+N}-\frac{w P}{a+N}\right) d \eta_{1} \\
& +\left(1-N-\frac{m P}{b+N}\right) d \eta_{1}
\end{aligned}
$$

where $M_{1}, M_{2}$ are positive numbers. Integrating both sides of the above inequality from 0 to $\tau_{n} \wedge T$ and taking the expectations give rise to

$$
\mathbf{E} V\left(N\left(\tau_{n} \wedge T\right), P\left(\tau_{n} \wedge T\right)\right) \leq V\left(N_{0}, P_{0}\right)+\left(M_{1}+M_{2}\right) T .
$$

Set $\Omega_{n}=\left\{\tau_{n} \leq T\right\}$ and by inequality (10), we get $\mathbf{P}\left(\Omega_{n}\right) \geq \varepsilon$. For every $\mu \in \Omega_{n}, N\left(\tau_{n}, \mu\right)$ and $P\left(\tau_{n}, \mu\right)$ equal either $n$ or $1 / n$, hence

$$
V\left(N\left(\tau_{n}, \mu\right), P\left(\tau_{n}, \mu\right)\right) \geq \min \left\{n-\ln n-1, \frac{1}{n}+\ln n-1\right\} .
$$

It then follows from (13) that

$$
\begin{aligned}
& V\left(N_{0}, P_{0}\right)+\left(M_{1}+M_{2}\right) T \\
& \quad \geq \mathbf{E}\left[1_{\Omega_{n}}(\mu) V\left(N\left(\tau_{n}\right), P\left(\tau_{n}\right)\right)\right] \\
& \quad \geq \varepsilon \min \left\{n-\ln n-1, \frac{1}{n}+\ln n-1\right\},
\end{aligned}
$$

where $1_{\Omega_{n}}$ is the indicator function of $\Omega_{n}$. Letting $n \rightarrow \infty$ leads to the contradiction $\infty>V\left(N_{0}, P_{0}\right)+\left(M_{1}+M_{2}\right) T=\infty$. This completes the proof. 


\subsection{Stochastic Boundedness}

Theorem 3. For any $\theta \in(0,1)$, there is a positive constant $C=$ $C(\theta)$, which is independent of initial value $\left(N_{0}, P_{0}\right) \in \mathbb{R}_{+}^{2}$, such that the solution of model (1) has the property that

$$
\limsup _{t \rightarrow \infty} \mathbf{E}|(N(t), P(t))|^{\theta} \leq C
$$

Proof. Define a function by

$$
V(N, P)=N^{\theta}+P^{\theta}, \quad(N, P) \in \mathbb{R}_{+}^{2} .
$$

For any $t>0$, using Itô formula gives that

$$
\begin{aligned}
\mathbf{L} V(N, P)= & r N^{\theta}\left(1-N-\frac{m}{b+N}-\frac{w P}{a+N}\right) \\
& +s \theta P^{\theta}\left(\frac{N}{a+N}-d-c P\right) \\
& +\frac{\alpha^{2}}{2} \theta(\theta-1) N^{\theta}\left(1-N-\frac{m}{b+N}-\frac{w P}{a+N}\right)^{2} \\
& +\frac{\beta^{2}}{2} \theta(\theta-1) P^{\theta}\left(\frac{N}{a+N}-d-c P\right)^{2},
\end{aligned}
$$

as $0<\theta<1$; then,

$$
\begin{aligned}
& \mathbf{L} V(N, P) \leq r \theta N^{\theta}(1-N)-\frac{\alpha^{2}}{2} \theta(1-\theta) N^{\theta} \\
& \times\left(1-N-\frac{m}{b+N}-\frac{w P}{a+N}\right)^{2}+s \theta P^{\theta} \frac{N}{a+N} \\
& -\frac{\beta^{2}}{2} \theta(1-\theta) P^{\theta}\left(\frac{N}{a+N}-d-c P\right)^{2} \\
& \leq r \theta N^{\theta}-\frac{\alpha^{2}}{2} \theta(1-\theta) N^{\theta} \\
& \times\left(\frac{m^{2}}{(b+N)^{2}}+(1-N)\left(1-N-\frac{2 m}{b+N}\right)-1\right) \\
& +s \theta P^{\theta}-\frac{\beta^{2}}{2} \theta(1-\theta) P^{\theta}\left(c^{2} P^{2}-2 c(1-d) P\right) \\
& =r \theta N^{\theta}-\frac{\alpha^{2}}{2} \theta(1-\theta) N^{\theta} \\
& \times\left(\frac{m^{2}}{(b+N)^{2}}+(1-N)\right. \\
& \left.\times\left(1-N-\frac{2 m}{b+N}\right)-1\right)+N^{\theta} \\
& +s \theta P^{\theta}-\frac{\beta^{2}}{2} \theta(1-\theta) P^{\theta}
\end{aligned}
$$

$$
\begin{aligned}
& \times\left(c^{2} P^{2}-2 c(1-d) P\right)+P^{\theta}-V(N, P) \\
\leq & C_{0}-V(N, P),
\end{aligned}
$$

where $C_{0}$ is an integer. Hence,

$$
\begin{aligned}
d V(N, P)= & \mathbf{L} V(N, P) d t \\
& +\alpha \theta N^{\theta}\left(1-N-\frac{m}{b+N}-\frac{w P}{a+N}\right) d \eta_{1} \\
& +\beta \theta P^{\theta}\left(\frac{N}{a+N}-d-c P\right) d \eta_{2} \\
\leq & \left(C_{0}-V(N, P)\right) d t \\
& +\alpha \theta N^{\theta}\left(1-N-\frac{m}{b+N}-\frac{w P}{a+N}\right) d \eta_{1} \\
& +\beta \theta P^{\theta}\left(\frac{N}{a+N}-d-c P\right) d \eta_{2} .
\end{aligned}
$$

Applying Itô formula yields

$$
\begin{aligned}
d\left(e^{t} V(N, P)\right)= & e^{t}(V(N, P) d t+d V(N, P)) \\
\leq & e^{t} C_{0} d t+e^{t} \alpha \theta N^{\theta} \\
& \times\left(1-N-\frac{m}{b+N}-\frac{w P}{a+N}\right) d \eta_{1} \\
& +e^{t} \beta \theta P^{\theta}\left(\frac{N}{a+N}-d-c P\right) d \eta_{2} .
\end{aligned}
$$

Using the sequence of stopping times $\left\{\tau_{n}\right\}_{n=1}^{\infty}$ defined in the proof of Theorem 2 and from (21), we have

$$
\begin{aligned}
& \mathbf{E}\left[e^{t \wedge \tau_{n}} V\left(N\left(t \wedge \tau_{n}\right), P\left(t \wedge \tau_{n}\right)\right)\right] \\
& \quad \leq V\left(N_{0}, P_{0}\right)+C_{0}\left(\mathbf{E} e^{t \wedge \tau_{n}}-1\right) \quad \forall t \geq 0 .
\end{aligned}
$$

Letting $n \rightarrow \infty$ in the latter inequality with a fact that $V\left(N\left(t \wedge \tau_{n}\right), P\left(t \wedge \tau_{n}\right)\right)>0$ and $0<e^{t \wedge \tau_{n}} \leq e^{t}$ a.s. and using Fatou's lemma, we obtain

$$
e^{t} \mathbf{E} V(N, P) \leq V\left(N_{0}, P_{0}\right)+C_{0}\left(e^{t}-1\right) .
$$

Therefore,

$$
\limsup _{t \rightarrow \infty} \mathbf{E} V(N(t), P(t)) \leq C .
$$

On the other hand, since

$$
|N, P|^{\theta} \leq \sqrt{2^{\theta}} \max \left\{N^{\theta}, P^{\theta}\right\} \leq \sqrt{2^{\theta}} V(N, P),
$$

we obtain

$$
\begin{gathered}
\limsup _{t \rightarrow \infty} \mathbf{E}|(N(t), P(t))|^{\theta} \\
\leq \sqrt{2^{\theta}} \limsup _{t \rightarrow \infty} \mathbf{E} V(N(t), P(t)) \\
\leq \sqrt{2^{\theta}} C \triangleq C(\theta) .
\end{gathered}
$$

The proof is complete. 
Theorem 4. The solutions of model (1) are stochastically ultimately bounded for any initial value $\left(N_{0}, P_{0}\right) \in \mathbb{R}_{+}^{2}$.

Proof. The solutions of model (1) will remain in $\mathbb{R}_{+}^{2}$ for all $t \geq$ 0 with probability one from Theorem 2 . And by Theorem 3 , let $\theta=1 / 2$; there is a $C_{1}>0$ such that

$$
\limsup _{t \rightarrow \infty} \mathbf{E}|(N(t), P(t))|^{1 / 2} \leq C_{1} .
$$

For any $\varepsilon>0$, set $X=C_{1}^{2} / \varepsilon^{2}$. Then by Chebyshev inequality,

$$
\mathbf{P}\{|(N(t), P(t))|>X\} \leq \frac{\mathbf{E}|(N(t), P(t))|^{1 / 2}}{\sqrt{X}} .
$$

That is,

$$
\limsup _{t \rightarrow \infty} \mathbf{P}\{|(N(t), P(t))|>X\} \leq \varepsilon
$$

This completes the proof.

Next, we study the asymptotic properties of the moment solutions of model (1).

Theorem 5. For any given $\theta \in(0,1)$, there is a $H=H(\theta)>$ 0 ; let $(N(t), P(t))$ be the solution of model (1) with any initial value $\left(N_{0}, P_{0}\right)$; then,

$$
\limsup _{t \rightarrow \infty} \frac{1}{t} \int_{0}^{t} \mathbf{E}\left(N^{\theta+2}(\tau)+P^{\theta+2}(\tau)\right) d \tau \leq H .
$$

Proof. Set $V(N, P): \mathbb{R}_{+}^{2} \rightarrow \mathbb{R}_{+}$, and from Theorem 3, we have

$\mathbf{L} V(N, P) \leq r \theta N^{\theta}-\frac{\alpha^{2}}{2} \theta(1-\theta) N^{\theta}$

$$
\begin{aligned}
& \times\left(N^{2}+\frac{m^{2}}{(b+N)^{2}}-\frac{2 m(1-N)}{b+N}-2 N\right) \\
& +s \theta P^{\theta}-\frac{\beta^{2}}{2} \theta(1-\theta) P^{\theta}\left(c^{2} P^{2}-2 c(1-d) P\right),
\end{aligned}
$$

so,

$$
\begin{aligned}
& \mathbf{L} V(N, P)+\frac{\alpha^{2} \theta(1-\theta)}{4} N^{\theta+2}+\frac{c^{2} \beta^{2} \theta(1-\theta)}{4} P^{\theta+2} \\
& \leq \quad r \theta N^{\theta}-\frac{\alpha^{2} \theta(1-\theta)}{4} N^{\theta+2} \\
& \quad+\alpha^{2} \theta(1-\theta) N^{\theta}\left(\frac{m(1-N)}{b+N}+N-\frac{m^{2}}{2(b+N)^{2}}\right) \\
& \quad+s \theta P^{\theta}-\frac{c^{2} \beta^{2} \theta(1-\theta)}{4} P^{\theta+2} \\
& \quad+c(1-d) \beta^{2} \theta(1-\theta) P^{\theta+1} \leq K
\end{aligned}
$$

where $K$ is a positive number. Then

$$
\begin{gathered}
\mathbf{L} V(N, P)+\frac{k^{2} \gamma^{2} \theta(1-\theta)}{4}\left(N^{\theta+2}+P^{\theta+2}\right) \\
\leq \mathbf{L} V(N, P)+\frac{\alpha^{2} \theta(1-\theta)}{4} N^{\theta+2} \\
+\frac{c^{2} \beta^{2} \theta(1-\theta)}{4} P^{\theta+2} \leq K
\end{gathered}
$$

where $k^{2} \gamma^{2}=\min \left\{\alpha^{2}, c^{2} \beta^{2}\right\}$; we can obtain

$$
\begin{aligned}
& \mathbf{E} V(N(t), P(t))+\frac{k^{2} \gamma^{2} \theta(1-\theta)}{4} \\
& \times \int_{0}^{t} \mathbf{E}\left(N^{\theta+2}(\tau)+P^{\theta+2}(\tau)\right) d \tau \\
& \quad \leq V\left(N_{0}, P_{0}\right)+K t .
\end{aligned}
$$

That is,

$$
\int_{0}^{t} \mathbf{E}\left(N^{\theta+2}(\tau)+P^{\theta+2}(\tau)\right) d \tau \leq \frac{4\left(V\left(N_{0}, P_{0}\right)+K t\right)}{k^{2} \gamma^{2} \theta(1-\theta)} .
$$

Set $H=4 K /\left(k^{2} \gamma^{2} \theta(1-\theta)\right)$; therefore,

$$
\limsup _{t \rightarrow \infty} \frac{1}{t} \int_{0}^{t} \mathbf{E}\left(N^{\theta+2}(\tau)+P^{\theta+2}(\tau)\right) d \tau \leq H .
$$

2.3. Stochastic Asymptotic Stability. From Theorem 2, model (1) has a global positive solution. By constructing some Lyapunov functions, we analyze stochastic asymptotic stability of the positive equilibria of the stochastic model. The equilibria of the stochastic model (1) we consider are solutions of the following model:

$$
\begin{gathered}
N\left(1-N-\frac{m}{b+N}-\frac{w P}{a+N}\right)=0, \\
P\left(\frac{N}{a+N}-d-c P\right)=0 .
\end{gathered}
$$

From (37), the positive equilibrium $(N, P)$ satisfies the equation

$$
\begin{gathered}
c N^{4}+(2 a c-c+c b) N^{3}+\left(a^{2} c-c(b-m)\right. \\
-2 a c(1-b)+w(1-d)) N^{2} \\
+\left(a^{2} c(b-1)-2 a c(b-m)\right. \\
+w b(1-d)-w a d) N \\
-a^{2} c(b-m)-w a b d=0, \\
N>\frac{a d}{1-d}>0 .
\end{gathered}
$$

As an example, we only give the proof of a positive equilibrium $\left(N^{*}, P^{*}\right)$ of model (1). 
Theorem 6. Let

$$
\theta=\frac{w\left(a+N^{*}\right)\left(r-\alpha^{2} N^{*}\right)}{a s+\beta^{2} a c P^{*}},
$$

and

$$
\begin{aligned}
A= & r\left(\frac{m}{b\left(b+N^{*}\right)}+\frac{w P^{*}}{a\left(a+N^{*}\right)}-1\right) \\
& +\frac{\alpha^{2} N^{*}}{2}\left(\frac{m}{b\left(b+N^{*}\right)}+\frac{w P^{*}}{a\left(a+N^{*}\right)}-1\right)^{2} \\
& +\frac{\beta^{2} a^{2} w P^{*}\left(r-\alpha^{2} N^{*}\right)}{2 a^{3}\left(a+N^{*}\right)\left(s+\beta^{2} c P^{*}\right)}, \\
B= & \frac{\alpha^{2} w^{2} N^{*}}{2 a^{2}} \\
+ & \frac{c w\left(a+N^{*}\right)\left(r-\alpha^{2} N^{*}\right)\left(\beta^{2} c P^{*}-2 s\right)}{2 a\left(s+\beta^{2} c P^{*}\right)}, \\
& C=\frac{\alpha^{2} m w N^{*}}{a b\left(b+N^{*}\right)}+\frac{\alpha^{2} w^{2} N^{*} P^{*}}{a^{2}\left(a+N^{*}\right)}
\end{aligned}
$$

if $A<0, C^{2}-4 A B<0$, then the equilibrium point $\left(N^{*}, P^{*}\right)$ of model (1) is stochastically asymptotically stable in $\mathbb{R}_{+}^{2}$; that is, for any initial value $\left(N_{0}, P_{0}\right)$, the solution of model (1) has the property that

$$
\lim _{t \rightarrow \infty} N(t)=N^{*}, \quad \lim _{t \rightarrow \infty} P(t)=P^{*},
$$

almost surely

Proof. Based on the theory of stability of stochastic differential equations [46], we only need to construct a Lyapunov function $V(U)$ satisfying $L V \leq 0$ and the identity holds if and only if $U=U^{*}$, where $U=U(t)$ is the solution of the stochastic differential equation (3) and $U^{*}$ is the equilibrium point of model (3).

Define Lyapunov functions

$$
\begin{gathered}
V_{1}(N)=N-N^{*}-N^{*} \ln \frac{N}{N^{*}}, \\
V_{2}(P)=P-P^{*}-P^{*} \ln \frac{P}{P^{*}},
\end{gathered}
$$

The nonnegativity of this function can be observed from $x-$ $1-\ln x \geq 0$ on $x>0$. If $(N(t), P(t)) \in \mathbb{R}_{+}^{2}$, applying Itô's formula, we have

$$
\begin{aligned}
\mathbf{L} V_{1}= & r\left(N-N^{*}\right)\left(1-N-\frac{m}{b+N}-\frac{w P}{a+N}\right) \\
& +\frac{\alpha^{2} N^{*}}{2}\left(1-N-\frac{m}{b+N}-\frac{w P}{a+N}\right)^{2}
\end{aligned}
$$

$$
\begin{gathered}
=\left[r\left(\frac{m}{\left(b+N^{*}\right)(b+N)}+\frac{w P^{*}}{\left(a+N^{*}\right)(a+N)}-1\right)\right. \\
+\frac{\alpha^{2} N^{*}}{2}\left(\frac{m}{\left(b+N^{*}\right)(b+N)}\right.
\end{gathered}
$$

$$
\begin{aligned}
& \left.\left.+\frac{w P^{*}}{\left(a+N^{*}\right)(a+N)}-1\right)^{2}\right] \\
& \times\left(N-N^{*}\right)^{2}+\frac{\alpha^{2} w^{2} N^{*}}{2(a+N)^{2}}\left(P-P^{*}\right)^{2} \\
& -\left[\frac{r w}{a+N}+\frac{\alpha^{2} w N^{*}}{a+N}\right. \\
& \left.\times\left(\frac{m}{\left(b+N^{*}\right)(b+N)}+\frac{w P^{*}}{\left(a+N^{*}\right)(a+N)}-1\right)\right] \\
& \times\left(N-N^{*}\right)\left(P-P^{*}\right) \\
& \leq\left[r\left(\frac{m}{b\left(b+N^{*}\right)}+\frac{w P^{*}}{a\left(a+N^{*}\right)}-1\right)\right. \\
& \left.+\frac{\alpha^{2} N^{*}}{2}\left(\frac{m}{b\left(b+N^{*}\right)}+\frac{w P^{*}}{a\left(a+N^{*}\right)}-1\right)^{2}\right] \\
& \times\left(N-N^{*}\right)^{2}+\frac{\alpha^{2} w^{2} N^{*}}{2 a^{2}}\left(P-P^{*}\right)^{2} \\
& +\frac{\alpha^{2} w N^{*}-r w}{a+N}\left(N-N^{*}\right)\left(P-P^{*}\right) \\
& +\left(\frac{\alpha^{2} m w N^{*}}{a b\left(b+N^{*}\right)}+\frac{\alpha^{2} w^{2} N^{*} P^{*}}{a^{2}\left(a+N^{*}\right)}\right)\left|N-N^{*}\right|\left|P-P^{*}\right| .
\end{aligned}
$$

Similarly, we obtain

$$
\begin{aligned}
& \mathbf{L} V_{2}= s\left(P-P^{*}\right)\left(\frac{N}{a+N}-d-c P\right) \\
&+\frac{\beta^{2} P^{*}}{2}\left(\frac{N}{a+N}-d-c P\right)^{2} \\
&= s\left(P-P^{*}\right)\left(\frac{a}{(a+N)\left(a+N^{*}\right)}\left(N-N^{*}\right)\right. \\
&+\frac{\beta^{2} P^{*}}{2}\left(\frac{\left.+c\left(P^{*}-P\right)\right)}{(a+N)^{2}\left(a+N^{*}\right)^{2}}\left(N-N^{*}\right)^{2}\right. \\
&+c^{2}\left(P-P^{*}\right)^{2} \\
&+\frac{2 a c}{(a+N)\left(a+N^{*}\right)} \\
&\left.\quad \times\left(N-N^{*}\right)\left(P-P^{*}\right)\right)
\end{aligned}
$$




$$
\begin{aligned}
= & \frac{\beta^{2} a^{2} P^{*}}{2(a+N)^{2}\left(a+N^{*}\right)^{2}}\left(N-N^{*}\right)^{2} \\
& +\left(\frac{\beta^{2} c^{2} P^{*}}{2}-s c\right)\left(P-P^{*}\right)^{2} \\
& +\frac{a s+a c \beta^{2} P^{*}}{(a+N)\left(a+N^{*}\right)}\left(N-N^{*}\right)\left(P-P^{*}\right) \\
\leq & \frac{\beta^{2} a^{2} P^{*}}{2 a^{2}\left(a+N^{*}\right)^{2}}\left(N-N^{*}\right)^{2} \\
& +\left(\frac{\beta^{2} c^{2} P^{*}}{2}-s c\right)\left(P-P^{*}\right)^{2} \\
& +\frac{a s+\beta^{2} a c P^{*}}{(a+N)\left(a+N^{*}\right)}\left(N-N^{*}\right)\left(P-P^{*}\right) .
\end{aligned}
$$

Define

$$
V(N, P)=V_{1}(N)+\theta V_{2}(P)
$$

and choose

$$
\theta=\frac{w\left(a+N^{*}\right)\left(r-\alpha^{2} N^{*}\right)}{a s+\beta^{2} a c P^{*}} .
$$

We compute

$$
\mathbf{L} V(N, P)
$$

$$
\begin{aligned}
= & \mathbf{L} V_{1}(N)+\mathbf{L} \theta V_{2}(P) \\
\leq & {\left[r\left(\frac{m}{b\left(b+N^{*}\right)}+\frac{w P^{*}}{a\left(a+N^{*}\right)}-1\right)\right.} \\
& +\frac{\alpha^{2} N^{*}}{2}\left(\frac{m}{b\left(b+N^{*}\right)}+\frac{w P^{*}}{a\left(a+N^{*}\right)}-1\right)^{2} \\
& \left.+\frac{\beta^{2} a^{2} w P^{*}\left(r-\alpha^{2} N^{*}\right)}{2 a^{2}\left(a+N^{*}\right)\left(a s+\beta^{2} a c P^{*}\right)}\right]\left(N-N^{*}\right)^{2} \\
& +\left[\frac{\alpha^{2} w^{2} N^{*}}{2 a^{2}}+\frac{c w\left(a+N^{*}\right)\left(r-\alpha^{2} N^{*}\right)\left(\beta^{2} c P^{*}-2 s\right)}{2\left(a s+\beta^{2} a c P^{*}\right)}\right] \\
& \times\left(P-P^{*}\right)^{2}+\left(\frac{\alpha^{2} m w N^{*}}{a b\left(b+N^{*}\right)}+\frac{\alpha^{2} w^{2} N^{*} P^{*}}{a^{2}\left(a+N^{*}\right)}\right)\left|N-N^{*}\right| \\
& \times\left|P-P^{*}\right|=A\left(N-N^{*}\right)^{2}+B\left(P-P^{*}\right)^{2} \\
& +C\left|N-N^{*}\right|\left|P-P^{*}\right| \cdot
\end{aligned}
$$

Let $\left|U-U^{*}\right|=\left(\left|N-N^{*}\right|,\left|P-P^{*}\right|\right)^{T}$; then, we have

$$
\mathbf{L} V(N, P) \leqslant \frac{1}{2}\left|U-U^{*}\right|^{T}\left(\begin{array}{cc}
2 A & C \\
C & 2 B
\end{array}\right)\left|U-U^{*}\right|
$$

Clearly, if $A<0$ and $C^{2}-4 A B<0$ hold, then the above inequality implies $\mathbf{L} V(N, P)<0$ along all trajectories in the first quadrant except $\left(N^{*}, P^{*}\right)$. Then the desired assertion (41) follows immediately.

\section{Numerical Simulations}

In this section, we perform extensive numerical simulations of model (1) to confirm the analytical results by referring to the method mentioned in Higham [47]. Next, we consider the discretization equations

$$
\begin{aligned}
N_{i+1}= & N_{i}+r N_{i}\left(1-N_{i}-\frac{m}{b+N_{i}}-\frac{w P_{i}}{a+N_{i}}\right) \Delta t \\
& +\alpha N_{i}\left(1-N_{i}-\frac{m}{b+N_{i}}-\frac{w P_{i}}{a+N_{i}}\right) \sqrt{\Delta t} \xi_{1 i} \\
& +\frac{\alpha^{2}}{2} N_{i}^{2}\left(1-N_{i}-\frac{m}{b+N_{i}}-\frac{w P_{i}}{a+N_{i}}\right)^{2}\left(\xi_{1 i}^{2}-1\right) \Delta t \\
P_{i+1}= & P_{i}+s P_{i}\left(\frac{N_{i}}{a+N_{i}}-d-c P_{i}\right) \Delta t \\
& +\beta P_{i}\left(\frac{N_{i}}{a+N_{i}}-d-c P_{i}\right) \sqrt{\Delta t} \xi_{2 i} \\
& +\frac{\beta^{2}}{2} P_{i}^{2}\left(\frac{N_{i}}{a+N_{i}}-d-c P_{i}\right)^{2}\left(\xi_{2 i}^{2}-1\right) \Delta t,
\end{aligned}
$$

where $\xi_{1 i}$ and $\xi_{2 i}(i=1,2, \ldots, n)$ are the Gaussian random variables $\mathbf{N}(0,1)$.

When choosing the environmental noise intensities $\alpha=$ $\beta=0$ (i.e., without noise), under the weak Allee effect, Figure 1 shows time-series plots and phase portraits of prey population $N$ and predator population $P$ of model (1) with initial value $\left(N_{0}, P_{0}\right)=(0.2,1.4)$ and other parameters values $r=1.5, s=0.6, a=0.5, b=0.6, c=0.1$, $d=0.12$, and $w=0.25$. In Figure 1(a), with the Allee effect $m=0.15$, one can see that a positive equilibrium point $\left(N^{*}, P^{*}\right)=(0.20589,1.71689)$ of model (1) is globally asymptotically stable, which illustrates the stable population distribution of both species. In Figure 1(b), increasing $m$ to $m=0.45$, a stable limit cycle occurs which indicates that prey population coexist with predator population with oscillator balance behavior.

Figure 2 shows time-series plots for model (1) with $r=$ $1.5, s=0.6, a=0.5, b=0.6, c=0.1, d=0.12, m=0.15$, and $w=0.25$ and initial condition $\left(N_{0}, P_{0}\right)=(0.2,1.4)$. The only difference between conditions of Figures 2(a) and 2(b) is that the values of environmental noise intensities $\alpha$ and $\beta$ are different. As pointed out in Theorem 6, if those conditions hold, then the positive equilibrium point $\left(N^{*}, P^{*}\right)$ is stochastically asymptotically stable in $\mathbb{R}_{+}^{2}$. In Figure $2(\mathrm{a})$, we choose $\alpha=\beta=0.5$ and the conditions of Theorem 6 are satisfied. That is to say, the equilibrium point $\left(N^{*}, P^{*}\right)$ is stochastically asymptotically stable. In Figure 2(b), choosing $\alpha=2.4$ and $\beta=0.8$, one can see that two species $N$ and $P$ will die out. In other words, when the environmental noise 

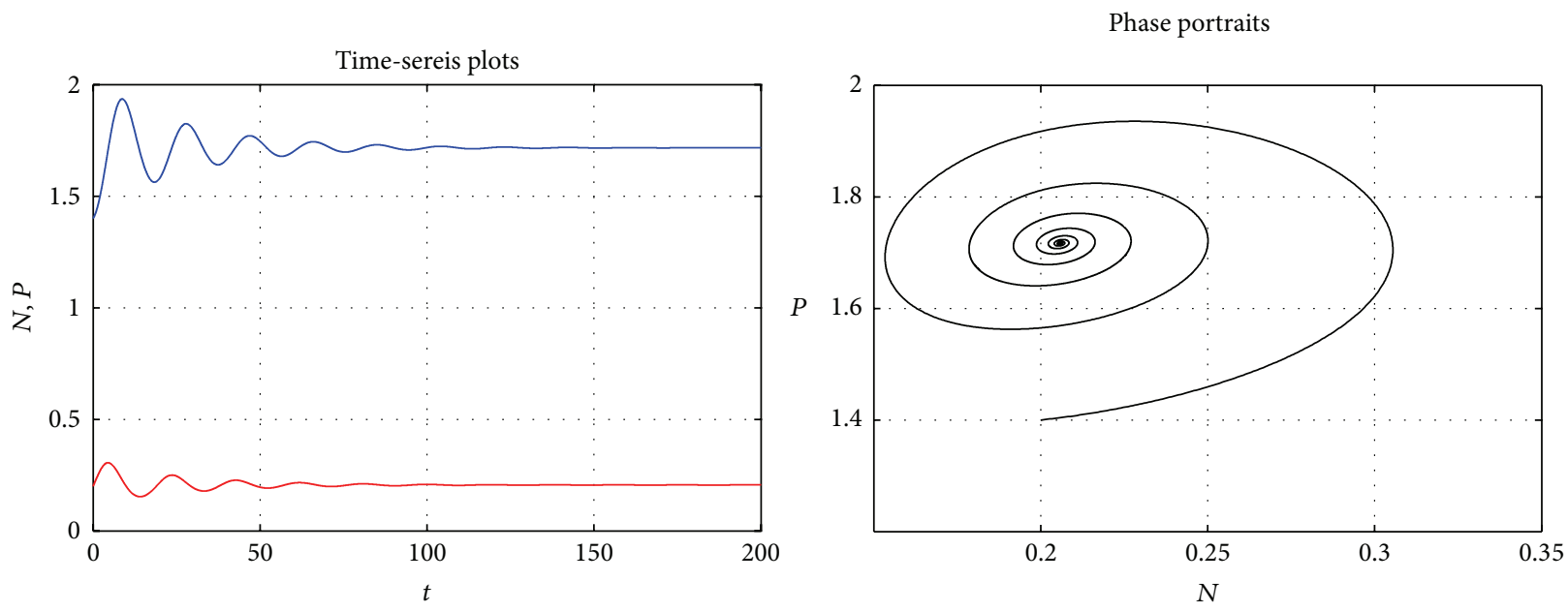

$-N$

(a)
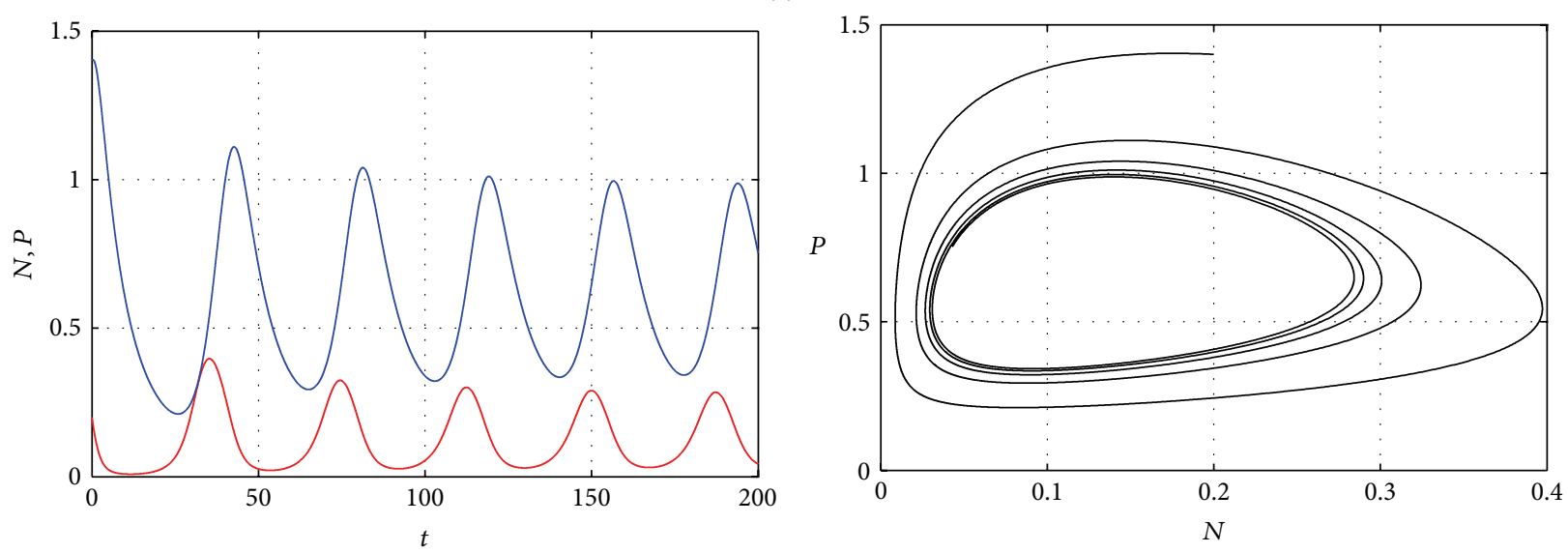

(b)

Figure 1: (left) Time-series plots; (right) phase portraits for model (1) without noise (i.e., $\alpha=\beta=0$ ) and with $r=1.5, s=0.6, a=0.5$, $b=0.6, c=0.1, d=0.12$, and $w=0.25$ and initial condition $\left(N_{0}, P_{0}\right)=(0.2,1.4)$. (a) $m=0.15$ and (b) $m=0.45$.

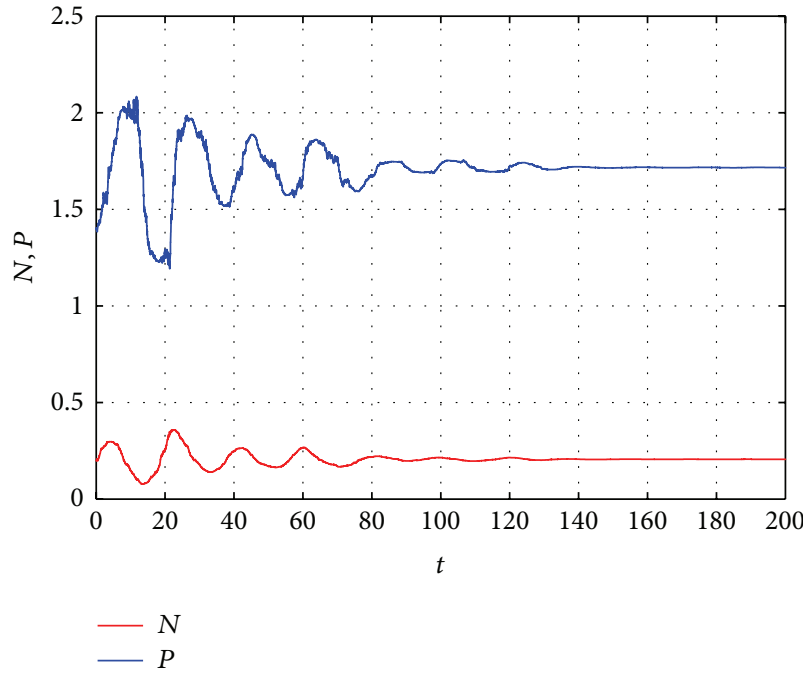

(a)

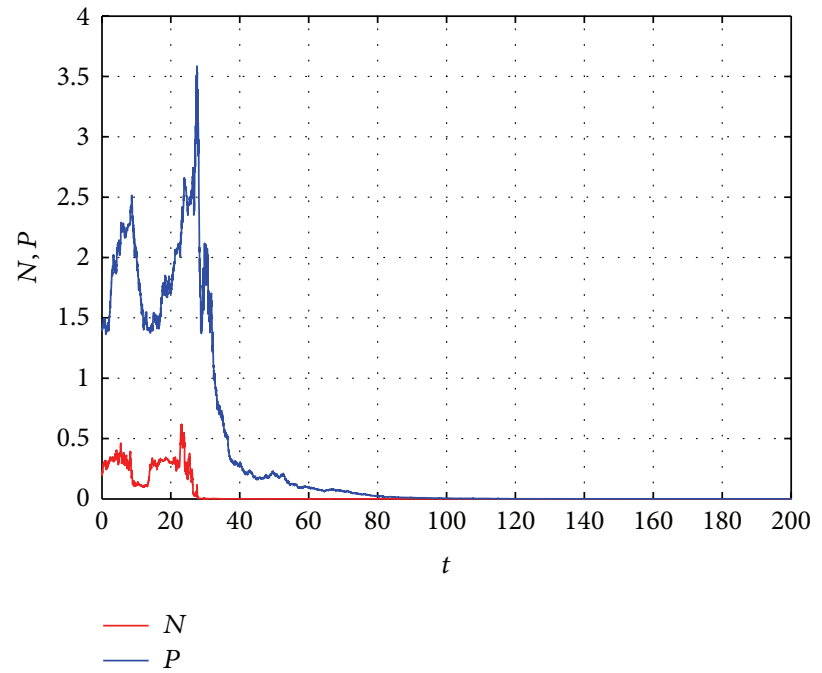

(b)

Figure 2: Time-series plots for model (1) with $r=1.5, s=0.6, a=0.5, b=0.6, c=0.1, d=0.12, m=0.15$, and $w=0.25$ and initial condition $\left(N_{0}, P_{0}\right)=(0.2,1.4)$. (a) $\alpha=\beta=0.5$ and (b) $\alpha=2.4$ and $\beta=0.8$. 
intensities are large and violate the conditions of Theorem 6, the positive equilibrium $\left(N^{*}, P^{*}\right)$ is no longer globally stable and not permanent.

By comparing Figure 1(a) and Figure 2(a), one can realize that if the noise is not strong, the stochastic perturbation does not cause sharp changes of the dynamics of model (1). However, when the environmental noise is sufficiently large (see Figure 2(b)), the noise can force the population to become extinct.

\section{Conclusions and Remarks}

In this paper, we propose a stochastic version of a modified Holling type-II predator-prey model with an Allee effect in prey population and take into account density-dependence of predator population. We perturb the model with respect to white noise around its intrinsic growth rate of the prey and predator population, respectively. By constructing suitable Lyapunov functions and applying Itô formula, some qualitative properties are given, such as the existence of global positive solutions, stochastic boundedness, and stochastic asymptotic stability which means that in the biological point of view, the community consisting of both prey and predator species is a stable biotic community where all species will coexist.

To our knowledge, the stability analysis of the stochastic Holling type-II predator-prey model with the Allee effect and density-dependence we illustrated here appears to be rare. And our complete analysis of the stochastic predator-prey model will give some suggestions to other models.

\section{Acknowledgments}

The author would like to thank the editor and the anonymous referees for their helpful comments and suggestions.

\section{References}

[1] J. D. Murray, Mathematical Biology. II. Spatial Models and Biomedical Applications, vol. 18 of Interdisciplinary Applied Mathematics, Springer, New York, NY, USA, Third edition, 2003.

[2] E. Renshaw, Modelling Biological Populations in Space and Time, vol. 11 of Cambridge Studies in Mathematical Biology, Cambridge University Press, Cambridge, UK, 1991.

[3] C. S. Holling, "The components of predation as revealed by a study of small-mammal predation of the European pine sawfly," The Canadian Entomologist, vol. 91, no. 5, pp. 293-320, 1959.

[4] C. S. Holling, "Some characteristics of simple types of predation and parasitism," The Canadian Entomologist, vol. 91, no. 7, pp. 385-398, 1959.

[5] G. T. Skalski and J. F. Gilliam, "Functional responses with predator interference: viable alternatives to the Holling type II model," Ecology, vol. 82, no. 11, pp. 3083-3092, 2001.

[6] X. Liu and L. Chen, "Complex dynamics of Holling type II Lotka-Volterra predator-prey system with impulsive perturbations on the predator," Chaos, Solitons \& Fractals, vol. 16, no. 2, pp. 311-320, 2003.
[7] B. Liu, Z. Teng, and L. Chen, "Analysis of a predator-prey model with Holling II functional response concerning impulsive control strategy," Journal of Computational and Applied Mathematics, vol. 193, no. 1, pp. 347-362, 2006.

[8] W. Ko and K. Ryu, "Qualitative analysis of a predator-prey model with Holling type II functional response incorporating a prey refuge," Journal of Differential Equations, vol. 231, no. 2, pp. 534-550, 2006.

[9] G. Jiang, Q. Lu, and L. Qian, "Complex dynamics of a Holling type II prey-predator system with state feedback control," Chaos, Solitons \& Fractals, vol. 31, no. 2, pp. 448-461, 2007.

[10] R. Yafia, F. El Adnani, and H. T. Alaoui, "Limit cycle and numerical similations for small and large delays in a predatorprey model with modified Leslie-Gower and Holling-type II schemes," Nonlinear Analysis: Real World Applications, vol. 9, no. 5, pp. 2055-2067, 2008.

[11] F. Rao, "Spatiotemporal pattern in a self- and cross-diffusive predation model with the Allee effect," Discrete Dynamics in Nature and Society, vol. 2013, Article ID 681641, 9 pages, 2013.

[12] B. Dennis, "Allee effects: population growth, critical density, and the chance of extinction," Natural Resource Modeling, vol. 3, no. 4, pp. 481-538, 1989.

[13] P. A. Stephens, W. J. Sutherland, and R. P. Freckleton, "What is the Allee effect?" Oikos, vol. 87, no. 1, pp. 185-190, 1999.

[14] G. Wang, X.-G. Liang, and F.-Z. Wang, "The competitive dynamics of populations subject to an Allee effect," Ecological Modelling, vol. 124, no. 2-3, pp. 183-192, 1999.

[15] S.-R. Zhou, Y.-F. Liu, and G. Wang, "The stability of predatorprey systems subject to the Allee effects," Theoretical Population Biology, vol. 67, no. 1, pp. 23-31, 2005.

[16] L. Berec, E. Angulo, and F. Courchamp, "Multiple Allee effects and population management," Trends in Ecology and Evolution, vol. 22, no. 4, pp. 185-191, 2007.

[17] P. Aguirre, E. González-Olivares, and E. Sáez, “Two limit cycles in a Leslie-Gower predator-prey model with additive Allee effect," Nonlinear Analysis: Real World Applications, vol. 10, no. 3, pp. 1401-1416, 2009.

[18] P. Aguirre, E. Gonález-Olivares, and E. S. Sáez, “Three limit cycles in a Leslie-Gower predator-prey model with additive Allee effect," SIAM Journal on Applied Mathematics, vol. 69, no. 5, pp. 1244-1262, 2009.

[19] W. Wang, Y. Cai, Y. Zhu, and Z. Guo, "Allee-effect-induced instability in a reaction-diffusion predator-prey model," Abstract and Applied Analysis, Article ID 487810, 10 pages, 2013.

[20] J. Wang, J. Shi, and J. Wei, "Predator-prey system with strong Allee effect in prey," Journal of Mathematical Biology, vol. 62, no. 3, pp. 291-331, 2011.

[21] I. Scheuring, "Allee effect increases the dynamical stability of populations," Journal of Theoretical Biology, vol. 199, no. 4, pp. 407-414, 1999.

[22] M.-H. Wang and M. Kot, "Speeds of invasion in a model with strong or weak Allee effects," Mathematical Biosciences, vol. 171, no. 1, pp. 83-97, 2001.

[23] A. Morozov, S. Petrovskii, and B.-L. Li, "Bifurcations and chaos in a predator-prey system with the Allee effect," Proceedings of the Royal Society B, vol. 271, no. 1546, pp. 1407-1414, 2004.

[24] J. Shi and R. Shivaji, "Persistence in reaction diffusion models with weak Allee effect," Journal of Mathematical Biology, vol. 52, no. 6, pp. 807-829, 2006.

[25] G. A. K. van Voorn, L. Hemerik, M. P. Boer, and B. W. Kooi, "Heteroclinic orbits indicate overexploitation in predator-prey 
systems with a strong Allee effect," Mathematical Biosciences, vol. 209, no. 2, pp. 451-469, 2007.

[26] J. Zu and M. Mimura, "The impact of Allee effect on a predatorprey system with Holling type II functional response," Applied Mathematics and Computation, vol. 217, no. 7, pp. 3542-3556, 2010.

[27] J. Wang, J. Shi, and J. Wei, "Dynamics and pattern formation in a diffusive predator-prey system with strong Allee effect in prey," Journal of Differential Equations, vol. 251, no. 4-5, pp. 1276-1304, 2011.

[28] R. Lin, S. Liu, and X. Lai, "Bifurcations of a predator-prey system with weak Allee effects," Journal of the Korean Mathematical Society, vol. 50, no. 4, pp. 695-713, 2013.

[29] R. Z. Khasminskii and F. C. Klebaner, "Long term behavior of solutions of the Lotka-Volterra system under small random perturbations," The Annals of Applied Probability, vol. 11, no. 3, pp. 952-963, 2001.

[30] X. Mao, G. Marion, and E. Renshaw, "Environmental Brownian noise suppresses explosions in population dynamics," Stochastic Processes and their Applications, vol. 97, no. 1, pp. 95-110, 2002.

[31] R. M. May, Stability and Complexity in Model Ecosystems, Princeton University Press, Princeton, NJ, USA, 2001.

[32] C. Ji, D. Jiang, and X. Li, "Qualitative analysis of a stochastic ratio-dependent predator-prey system," Journal of Computational and Applied Mathematics, vol. 235, no. 5, pp. 1326-1341, 2011.

[33] H. Malchow, F. M. Hilker, and S. V. Petrovskii, "Noise and productivity dependence of spatiotemporal pattern formation in a prey-predator system," Discrete and Continuous Dynamical Systems. Series B, vol. 4, no. 3, pp. 705-711, 2004.

[34] X. Mao, "Stochastic stabilization and destabilization," Systems \& Control Letters, vol. 23, no. 4, pp. 279-290, 1994.

[35] C. A. Braumann, "Variable effort harvesting models in random environments: generalization to density-dependent noise intensities," Mathematical Biosciences, vol. 177-178, pp. 229-245, 2002.

[36] M. Bandyopadhyay and C. G. Chakrabarti, "Deterministic and stochastic analysis of a nonlinear prey-predator system," Journal of Biological Systems, vol. 11, no. 2, pp. 161-172, 2003.

[37] M. Bandyopadhyay and J. Chattopadhyay, "Ratio-dependent predator-prey model: effect of environmental fluctuation and stability," Nonlinearity, vol. 18, no. 2, pp. 913-936, 2005.

[38] X. Mao, C. Yuan, and J. Zou, "Stochastic differential delay equations of population dynamics," Journal of Mathematical Analysis and Applications, vol. 304, no. 1, pp. 296-320, 2005.

[39] R. Rudnicki and K. Pichór, "Influence of stochastic perturbation on prey-predator systems," Mathematical Biosciences, vol. 206, no. 1, pp. 108-119, 2007.

[40] M. Bandyopadhyay, T. Saha, and R. Pal, "Deterministic and stochastic analysis of a delayed allelopathic phytoplankton model within fluctuating environment," Nonlinear Analysis: Hybrid Systems, vol. 2, no. 3, pp. 958-970, 2008.

[41] C. Ji, D. Jiang, and N. Shi, "Analysis of a predator-prey model with modified Leslie-Gower and Holling-type II schemes with stochastic perturbation," Journal of Mathematical Analysis and Applications, vol. 359, no. 2, pp. 482-498, 2009.

[42] F. Rao, W. Wang, and Z. Li, "Spatiotemporal complexity of a predator-prey system with the effect of noise and external forcing," Chaos, Solitons \& Fractals, vol. 41, no. 4, pp. 1634-1644, 2009.

[43] A. Maiti and S. Pathak, "A modified Holling-Tanner model in stochastic environment," Nonlinear Analysis: Modelling and Control, vol. 14, no. 1, pp. 51-71, 2009.
[44] M. Liu and K. Wang, "Global stability of a nonlinear stochastic predator-prey system with Beddington-DeAngelis functional response," Communications in Nonlinear Science and Numerical Simulation, vol. 16, no. 3, pp. 1114-1121, 2011.

[45] X. Wang, H. Huang, Y. Cai, and W. Wang, "The complex dynamics of a stochastic predator-prey model," Abstract and Applied Analysis, vol. 2012, Article ID 401031, 24 pages, 2012.

[46] X. Mao, Stochastic Differential Equations and Their Applications, Horwood Publishing Series in Mathematics \& Applications, Horwood, Chichester, UK, 1997.

[47] D. J. Higham, "An algorithmic introduction to numerical simulation of stochastic differential equations," SIAM Review, vol. 43, no. 3, pp. 525-546, 2001. 


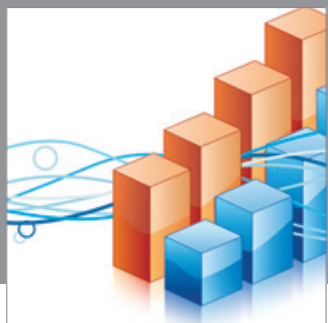

Advances in

Operations Research

mansans

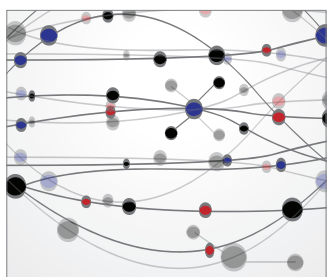

The Scientific World Journal
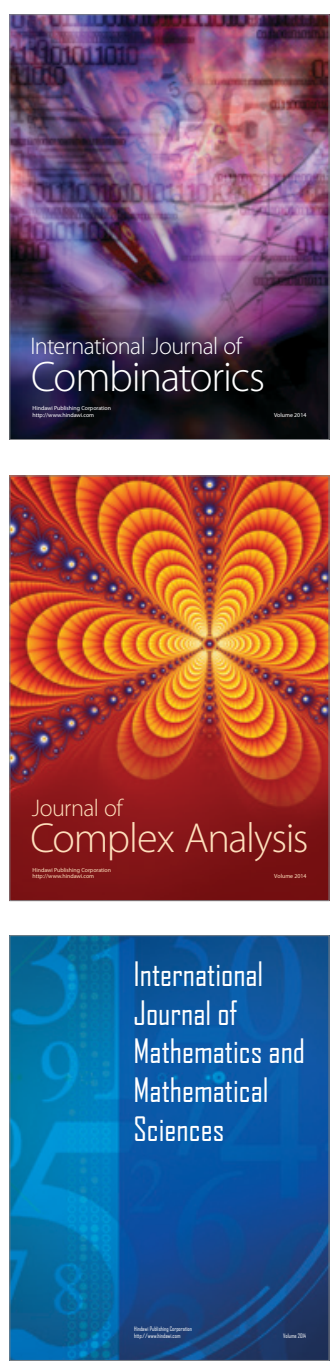
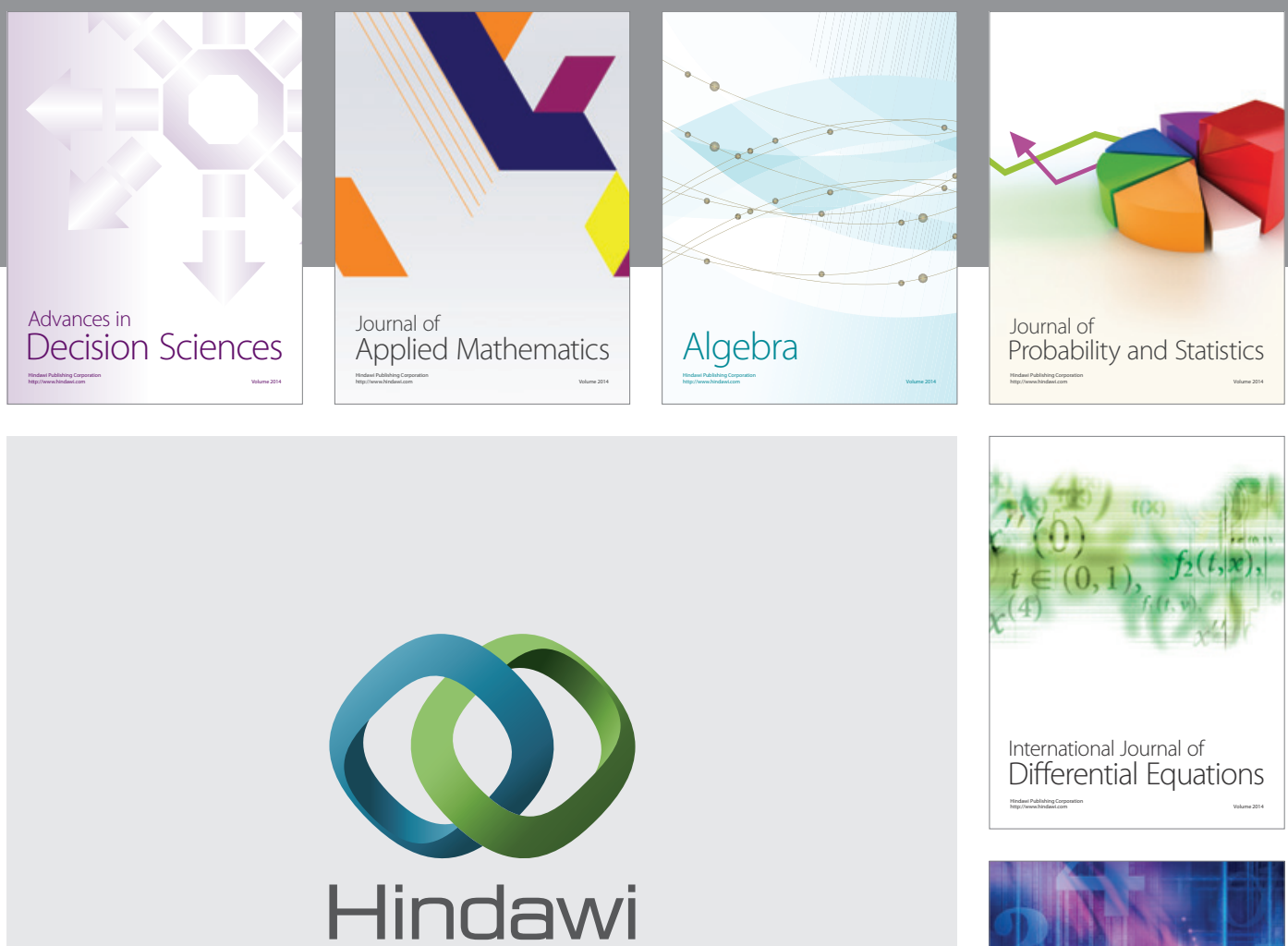

Submit your manuscripts at http://www.hindawi.com
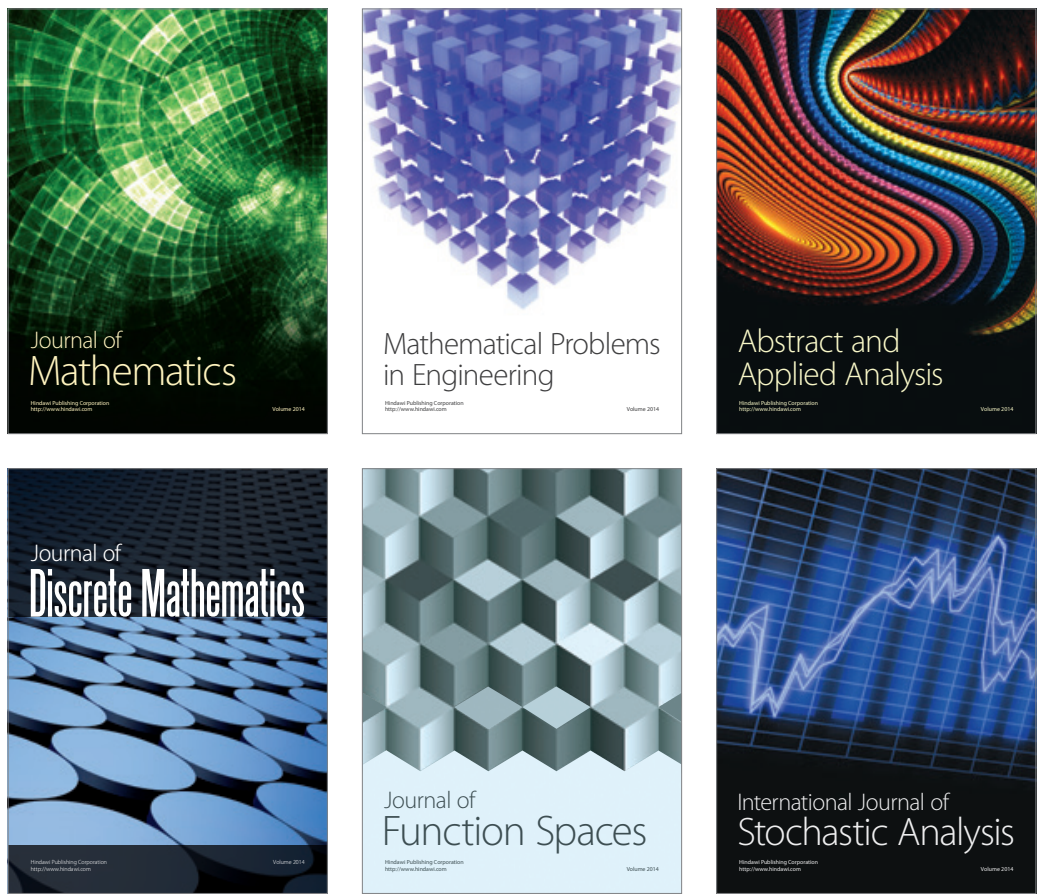

Journal of

Function Spaces

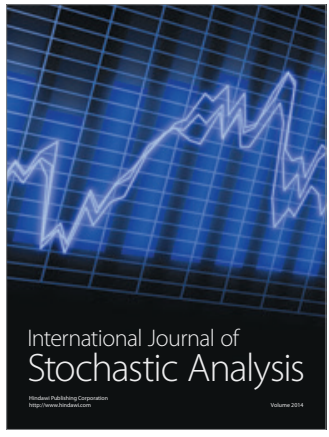

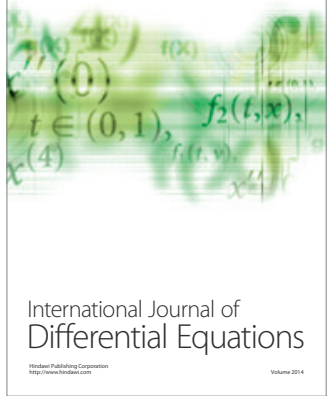
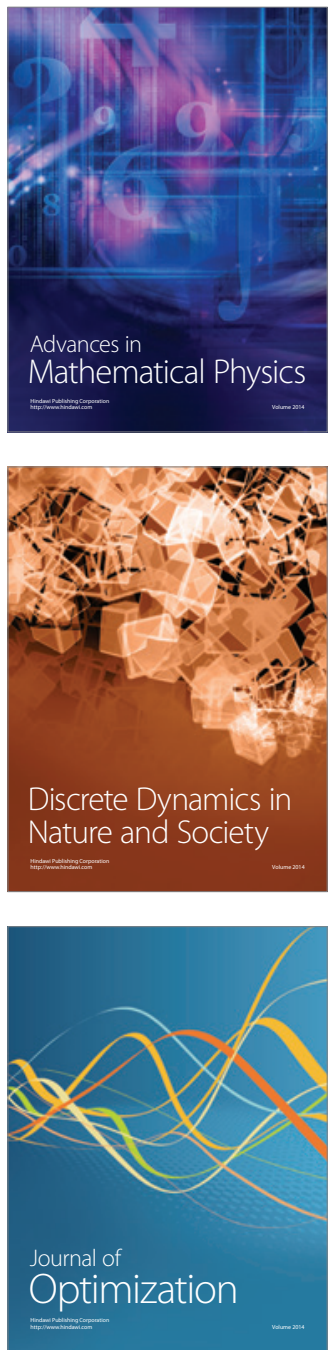